\title{
Effect of the Dietary Inclusion of Soybean Components on the Innate Immune System in Zebrafish
}

\author{
Pamela Fuentes-Appelgren,,2 Rafael Opazo,, Luis Barros, Carmen G. Feijoó, ${ }^{3}$ \\ Victoria Urzúa, and Jaime Romero ${ }^{1}$
}

\begin{abstract}
Some components of plant-based meals, such as saponins and vegetal proteins, have been proposed as inducers of intestinal inflammation in some fish. However, the molecular and cellular bases for this phenomenon have not been reported. In this work, zebrafish were used as a model to evaluate the effects of individual soybean meal components, such as saponins and soy proteins. Zebrafish larvae fed a fish meal feed containing soy components were assessed according to low and high inclusion levels. The granulocytes associated with the digestive tract and the induction of genes related to the immune system were quantitated as markers of the effects of the dietary components. A significant increase in the number of granulocytes was observed after feeding fish diets containing high saponin or soy protein contents. These dietary components also induced the expression of genes related to the innate immune system, including myeloid-specific peroxidase, as well as the complement protein and cytokines. These results reveal the influence of dietary components on the stimulation of the immune system. These observations could be significant to understanding the contributions of saponin and soy protein to the onset of enteritis in aqua-cultured fish, and this knowledge may aid in defining the role of the innate immune system in other inflammatory diseases involving dietary components in mammals.
\end{abstract}

\section{Introduction}

$\mathbf{T}$ HE DIGESTIVE TRACT is a critical location for the combined interactions among the host, the microbiota, and the elements ingested in the diet. ${ }^{1,2}$ In this compartment, the high levels of metabolic activity that occur are essential for several host attributes, including nutrition and immunity., ${ }^{2,3}$ The primary purpose of the digestive tract is to digest foodstuffs into molecules suitable for absorption by the various transport mechanisms of epithelial border cells. ${ }^{4,5}$ Some foodstuffs can alter these functions, especially when dietary changes are unavoidable. ${ }^{6-8}$ This phenomenon is particularly relevant in the case of farmed fish because their current diets include a greater proportion of crop ingredients, which are used to replace increasingly expensive fish meal. ${ }^{9-11}$ The inclusion of plant-based ingredients may have deleterious effects on fish growth and performance, especially in carnivorous fish such as salmonids, ${ }^{8,10}$ and these effects can be related to enteritis syndrome. ${ }^{12}$ This pressure for replacement has generated interest in studies focused in the impact of these ingredients on the health of the fish digestive system and its relationship with the immune system. ${ }^{8}$
Because of its availability, soybean meal (SBM) represents an alternative protein source for use in feed production. ${ }^{12}$ SBM contains $462-562 \mathrm{~g} / \mathrm{kg}$ crude protein and an appropriate amino acid profile. ${ }^{12}$ However, the problem with the consumption of a plant-based diet that includes SBM products is the induction of an inflammatory response in the digestive tract of a broad spectrum of fish (enteritis). This disorder is characterized by changes in absorptive cells, the increased presence of inflammatory cells, endocytic blocking, the shortening of villi, and the disruption of microvilli. This disorder may at least partially explain the negative effects of SBM products on growth parameters and feed utilization. ${ }^{13-16}$ Immunological mechanisms are likely involved in the underlying pathology, but the precise causes of the inflammatory process have not yet been clarified. It is accepted that these effects are mediated, at least in part, by antinutritional factors, such as soyasaponins (SAPs), which disrupt the intestinal barrier by altering membrane permeability. ${ }^{17-19}$ Saponins are glycosides produced by more than 100 families of plants, such as soy, pea, sunflower, and lupin. Soybeans generally contain saponins in the range of $1-5 \mathrm{~g} / \mathrm{kg}$, and the levels in soybeans are generally higher than in other

\footnotetext{
${ }^{1}$ Laboratorio de Biotecnología, Instituto de Nutrición y Tecnología de los Alimentos (INTA), Universidad de Chile, Santiago, Chile.

${ }^{2}$ Biotechnology PhD Program, University of Santiago of Chile, Santiago, Chile.

${ }^{3}$ Department of Biological Sciences, University Andres Bello, Santiago, Chile.
} 
common feedstuffs. ${ }^{20}$ Saponins are amphipathic molecules containing a hydrophobic steroidal or triterpenoid aglycone to which one or more hydrophilic sugar chains are attached. The inclusion of SAPs into fish diets has been associated with morphological changes and inflammation of the intestinal epithelium in Salmo salar, Cyprinus carpio, and Paralichthys olivaceus. ${ }^{15,19,21}$

The current data on SBM use in salmonids indicate deleterious effects at moderate-to-high levels of its inclusion in their diet; however, the factors causing these negative effects remain unknown. Knudsen et al. ${ }^{18}$ incorporated a crude ethanol extract from soybeans into a salmon diet and concluded that both soy saponins and an unidentified compound were likely contributors to enteritis in salmon. The immunological responses induced by saponin inclusion in $S$. salar have been recently revealed by Kortner et al. ${ }^{22}$ In this study, saponin was included into different plant-based diets, and the transcriptomic response was analyzed using microarray technologies. The combination of saponin with pea concentrate protein produced the highest enteritis levels as well as the upregulation of several genes, including cytokines, $\mathrm{NF} \kappa \mathrm{B}$ and TNF- $\alpha$-related genes, and regulators of T-cell function.

Similarly, Chikwati et al. ${ }^{23}$ evaluated saponin supplementation in combination with several plant-based proteins. In a diet containing pea protein concentrate, saponin supplementation induced dramatic effects that were similar to the typical effects induced by SBM diets. In contrast, dietary SAP supplementation $(20 \%-30 \%$ SBM) had minor negative effects when combined with maize gluten or sunflower meal. Therefore, these studies reveal the importance of protein source in the modulation of enteritis levels.

Glycinin and $\beta$-conglycinin, the major storage proteins in soy isolates, have been investigated using various animal models, including mice, swine, and calves. ${ }^{24-26}$ Collins et al. ${ }^{27}$ studied the inclusion of soybean, pea, and canola meals and their protein concentrates on the growth of rainbow trout but found only minor growth with SBM and canola meal; soybean and canola protein concentrates did not show alterations in the growth parameters. These results suggest that plant-based proteins may be involved in the induction of inflammatory signals. It is well known that soy proteins have immunogenic properties, ${ }^{28}$ but the occurrence of this phenomenon does not establish whether soy proteins are immunogenic in fish.

It is clear that the etiology of the SBM-induced enteritis process needs to be better characterized. More knowledge about the early development of enteritis will contribute to the elucidation of the mechanisms underlying this disorder and may shed light on other food-sensitive enteropathies, such as human celiac disease, which is an intestinal disorder associated with vegetal proteins. The zebrafish (Danio rerio) is a commonly used model organism in developmental, molecular, and toxicological studies. It has been proposed as a model for immunological studies, and more recently, it has been explored as a model for nutrition ${ }^{29}$ for inflammatory disorders of the digestive tract. ${ }^{30}$ Sissener et al. ${ }^{31}$ used zebrafish in a feeding trial study to assess their use as a model organism for feed ingredient safety assessments.

In this study, the zebrafish was used as a model to assess the effect of soybean components on the immune system. The purpose was to evaluate whether SAP or soy protein isolate (SPI) supplementation in a fish meal diet might affect the innate immune system of zebrafish by promoting granulocyte activation and inducing inflammatory signals, as assessed at the level of gene expression.

\section{Materials and Methods}

\section{Zebrafish husbandry and feeding}

Zebrafish were maintained and raised in our facility according to standard protocols. ${ }^{32}$ The Tab5 (wild type) strain and Tg(mpx:GFP) $)^{i 14}$ (ZFIN ID: ZDB-GENO-070118-1) line of zebrafish were used in this study. All embryos were collected by natural spawning, staged according to Kimmel et al., ${ }^{33}$ and raised at $28.5^{\circ} \mathrm{C}$ in the $\mathrm{E} 3$ medium $(5 \mathrm{mM} \mathrm{NaCl}, 0.17 \mathrm{mM} \mathrm{KCl}$, $0.33 \mathrm{mM} \mathrm{CaCl}, 0.33 \mathrm{mM} \mathrm{MgSO}_{4}$ without methylene blue, equilibrated to $\mathrm{pH}$ 7.0) in Petri dishes, as described previously. ${ }^{34}$ The embryonic and larval ages are expressed in days post-fertilization (dpf). All animals subjected to experimentation were anesthetized with tricaine (Sigma), and all procedures complied with the guidelines of the Animal Ethics Committees of the Universidad de Chile.

After hatching, the larvae were rinsed twice and transferred to six-well plates. Beginning at $5 \mathrm{dpf}$, the larvae were provided with experimental diets twice daily (Table 1). Each dietary treatment was performed at least in triplicate. The powdered feed was left over the wells for $1 \mathrm{~h}$, after which time the larvae were rinsed and transferred to a new plate. The treatment was completed at $10 \mathrm{dpf}$ (after 5 days of feeding), and gene expression analysis and granulocyte quantification were performed. As a reference, a group of control zebrafish was fed a diet consisting of fish meal $(100 \%$ of the protein was provided by fish meal). The experimental diets corresponded

Table 1. Experimental Diets for Feeding Zebrafish Larvae

\begin{tabular}{|c|c|c|c|c|c|}
\hline Ingredients $(\mathrm{g} / \mathrm{kg})$ & Control diet & SPI (low) diet ${ }^{\mathrm{a}}$ & SPI (high) diet ${ }^{\mathrm{b}}$ & SAP (low) diet ${ }^{\mathrm{c}}$ & SAP (high) diet ${ }^{\mathrm{d}}$ \\
\hline Fish meal & 640 & 426 & 284 & 640 & 632 \\
\hline Soy protein isolate & - & 137 & 311 & - & - \\
\hline SAP & - & - & - & 6.6 & 11 \\
\hline Wheat meal & 190 & 126 & 85 & 189 & 188 \\
\hline Vitamins and minerals & 2.7 & 2.7 & 2.7 & 2.7 & 2.7 \\
\hline Total protein content & 484 & 421 & 478 & 483 & 478 \\
\hline
\end{tabular}

a The supplementation level of soy protein approximated a level provided by a 30\% SBM dietary inclusion.

${ }^{b}$ The supplementation level of soy protein approximated a level provided by a $50 \%$ SBM dietary inclusion.

${ }^{c}$ The supplementation level of SAP approximated a level provided by a 30\% SBM dietary inclusion.

${ }^{\mathrm{d}}$ The supplementation level of SAP approximated a level provided by a 50\% SBM dietary inclusion.

$\mathrm{SBM}$, soybean meal; SPI, soy protein isolate; SAP, soyasaponin. 
to diets supplemented with SAP or SPIs (Gushen Biological Technology Group Co., Ltd.). These components were incorporated at two levels. The low-SPI or low-SAP diets corresponded to a supplementation with SAP or soy protein at approximately the level provided by $30 \%$ SBM dietary inclusion (Table 1), with 70\% of the protein from fish meal and $30 \%$ from soy protein. The high-SPI or high-SAP diets corresponded to supplementation with SAP or soy protein at approximately the level provided by $50 \%$ SBM dietary inclusion. In high-SPI diet, $50 \%$ of the protein was from fish meal and $50 \%$ was from soy protein. The "low" diets mimicked the current levels of SBM in fishfeed, whereas the "high" diets corresponded to an experimental approach to reveal the effects of the individually supplemented components upon the induction of inflammatory signals.

The fraction of larvae ingesting the experimental diets was evaluated. For each diet, 100 larvae were assessed in groups containing 20 fish and were fed for 5 days as described previously. After feeding, the larvae were examined under a stereomicroscope to determine the fraction of larvae containing food in the digestive tract. During the experiment, $\sim 50 \%$ of the larvae contained ingested food in their digestive tracts. A representative assay is shown in Supplementary Figures S1 and S2 (Supplementary Data are available online at www.liebertpub.com/zeb). Considering this result, only the larvae exhibiting feed within their guts were analyzed in the experiments involving SAP or SPI supplementation.

In preliminary studies, the control diet $(100 \%$ of the protein from fish meal) was compared with the Sera micron zebrafish diet, which is commonly used at this growth stage. The number of granulocytes that were associated with the digestive tract and the expression of inflammation-related genes were evaluated. For each diet, 120 larvae were assessed in groups containing 20 fish and were fed for 5 days as described previously. The results were derived from the fraction of larvae that ingested the feed. The results indicated no significant differences in the number of granulocytes associated with the digestive tracts of the larvae fed with these diets (Supplementary Fig. S3). However, a slight stimulation of the genes related to the immune system was observed in larvae fed with the Sera micron commercial diet (Supplementary Fig. S4).

\section{Sudan Black staining of granulocytes}

To identify the granulocytes in the zebrafish larvae, Sudan Black staining was performed, as described previously. ${ }^{3}$ The larvae were anesthetized and mounted as described. ${ }^{3}$ The larvae were fixed for $90 \mathrm{~min}$ in $4 \%$ glutaraldehyde buffer for $30 \mathrm{~min}$ at room temperature and then were stained with $0.18 \%$ Sudan Black, followed by extensive washing in 70\% ethanol. Considering that in the stomachless larvae, the absorptive function is performed along the digestive tract, and the absorbed molecules (proteins) are degraded intracellularly, ${ }^{35}$ the granulocytes were observed for the purposes of this study using a lateral view of the tail, which contains the entire digestive tract. The images were captured with a Moticam 2500 camera connected to an SMZ168 Motic stereomicroscope. The images were then processed using Image Analyzer 1.32 software. The granulocytes associated with each fish's digestive tract were quantified. These results were processed using the SPSS 8.0 program by performing a one-way analysis of vari- ance (ANOVA) to identify significant differences between the experimental groups. To determine the significant differences between groups, Dunnett's multiple comparison was used.

\section{Detection of neutrophils in the digestive tract using Tg(mpx:GFP) ${ }^{i 14}$ line zebrafish}

To determine the location of neutrophils in the digestive tract of fish, a transgenic fish line was used $\operatorname{Tg}(m p x: G F P)^{i 114} .36$ This line has been proposed as an in vivo model for genetic analysis of the inflammatory response and expresses green fluorescent protein (GFP) under the neutrophil-specific myeloperoxidase promoter. Cells expressing GFP were located in zebrafish sections using an anti-GFP antibody (cat: A11122; Invitrogen) and an immunohistochemistry protocol described by Westerfield et al. ${ }^{32}$ The detection of neutrophils was performed on zebrafish fed with the control diet or experimental diets (high-SPI, high-SAP), as described previously. The secondary antibody was goat anti-rabbit IgG-HR Peroxidase (cat: A8275; Sigma), and ImmPACT DAB PeroxidaseSubstrate (3,3'-Diaminobenzidine) was used to reveal the brown color (Vector Laboratories). Sections lacking the primary antibody were used as negative controls. After completion of the feeding period, the fish were fixed with $4 \%$ paraformaldehyde in phosphate-buffered saline for $45 \mathrm{~min}$, and the larvae were transferred finally to $100 \%$ methanol (MeOH) and stored at $-20^{\circ} \mathrm{C}$ overnight. To complement the histological analysis, sagittal sections of the zebrafish larvae were stained with hematoxylin and eosin.

\section{Histology of fish sections}

Whole zebrafish were embedded in Epon resin for electron microscopy. Sagittal sections were constructed with a slice thickness of $2 \mu \mathrm{m}$ by the Leica RM2135 microtome. Finally, the sections were stained with toluidine blue for $15 \mathrm{~s}$ for observation under the microscope.

\section{Gene expression analysis}

To study the immunogenic properties of SAP or SPI incorporation into the high-SPI and high-SAP diets, a set of genes related to the innate immune system was assessed. These genes were myeloid-specific peroxidase (mpx), complement component $\mathrm{c} 3 \mathrm{~b}(\mathrm{c} 3 b)$, lysozyme (lyz), serum amyloid A (saa), and the cytokines interleukin 1, beta (illb), and tumor necrosis factor a $(\operatorname{tnfa})$. Their relative expressions were determined using the larvae fed the control diet $(100 \%$ protein of fish meal) as the reference condition; this reference was compared to the treatment condition, which corresponded to the larvae fed diets supplemented with SAP or SPI at high levels of supplementation. For each diet, three replicates of 120 larvae were assessed in groups containing 20 fish and were fed for 5 days as described previously. Only larvae that ingested feed were considered for further analysis. Total RNA was extracted from pools containing five larvae using TriPure reagent (Roche) according to the manufacturer's instructions and was then stored at $-80^{\circ} \mathrm{C}$. Subsequently, cDNA was obtained using the ImProm-II Reverse Transcription system (Promega) according to the manufacturer's instructions with random hexamer primers. Each cDNA sample was assessed in quadruplicate (four quantitative polymerase chain [qPCR] reactions); primers and qPCR conditions appear in Table 2 as described by Rawls et al. ${ }^{1}$ and specificities were 


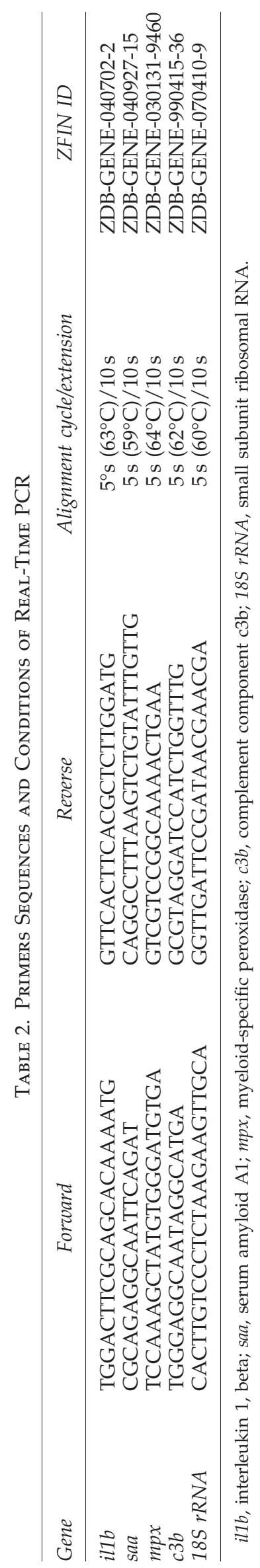

validated using melting curves. The mean $\mathrm{Ct}$ values from each sample were normalized against the mean $\mathrm{Ct}$ value of a reference gene (ribosomal RNA, 18S, housekeeping gene) as previously described. ${ }^{1}$ The relative quantification of each gene was obtained using the Pfaffl method and REST 2009 software (Qiagen). This software includes a statistical test to determine the accuracy of the relative expression, which is complex because the ratio distributions do not have a standard deviation. The REST 2009 software overcomes this

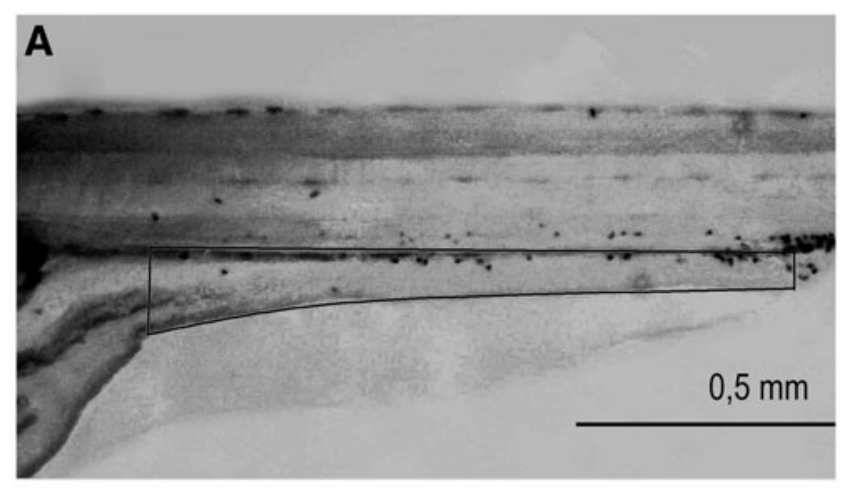

B

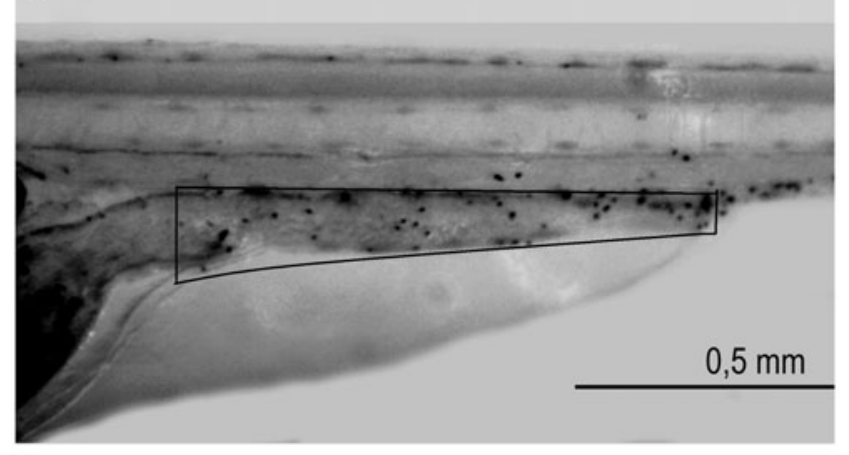

C

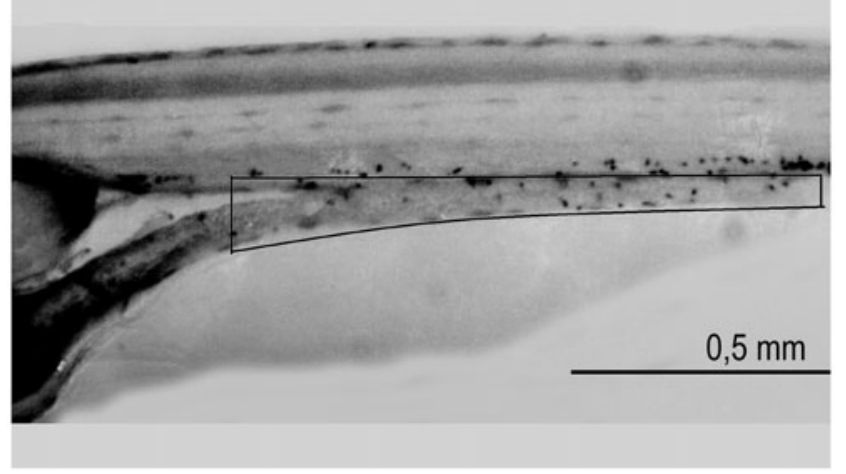

FIG. 1. Larvae after staining with Sudan Black to reveal the granulocyte number after being fed different diets. Visualization of granulocytes localized close to the digestive tract after Sudan Black staining. Larvae fed the control diet $(100 \%$ fish meal based) are shown in (A); larvae fed the high-soy protein isolate (SPI) diet in (B); and larvae fed the highsoyasaponin (SAP) diet in $(\mathrm{C})$. The region marked with the black rectangle indicates the area evaluated for the granulocyte count. The images were taken with a camera Moticam 2500 connected to an SMZ168 Motic stereomicroscope. 
limitation by using a simple statistical randomization test that analyzes the permuted expression data rather than the raw $\mathrm{Ct}$ values input by the user. ${ }^{37}$

\section{Results}

\section{Effect of dietary supplementation on the number of granulocytes associated with the digestive tract}

At $5 \mathrm{dpf}$, triplicate groups of fish were fed a dry microparticulate feed $(20 \mu \mathrm{m})$; the dietary treatment was completed at $10 \mathrm{dpf}$. Then, the larvae were stained with Sudan Black to examine the number of granulocytes around the digestive tract. The images captured corresponded to lateral views of the larvae's tails to visualize the granulocytes along the body. In particular, granulocytes were observed in the sections close to the digestive tract. In Figure 1, the characteristic black staining that identifies the granulocytes can be observed. Figure 1A shows a larva fed the control diet; few granulocytes $($ mean $=16)$ are located near the digestive tract. In contrast, Figure $1 \mathrm{~B}$ presents a larva fed the high-SPI diet, in which the number of granulocytes in the analyzed area was increased $($ mean $=23)$. This high number of granulocytes was also observed in larvae fed the high-SAP diets (Fig. 1C).

The number of granulocytes in the larvae fed the control diet was compared with the numbers observed in the larvae fed the plant-based diets (low- and high-SAP or low- and high-SPI diets). Figure 2 presents the means and distribution obtained after statistical analysis, indicating that larvae fed low-SAP or low-SPI diets showed no significant differences from the control group. In contrast, larvae fed high-SAP or

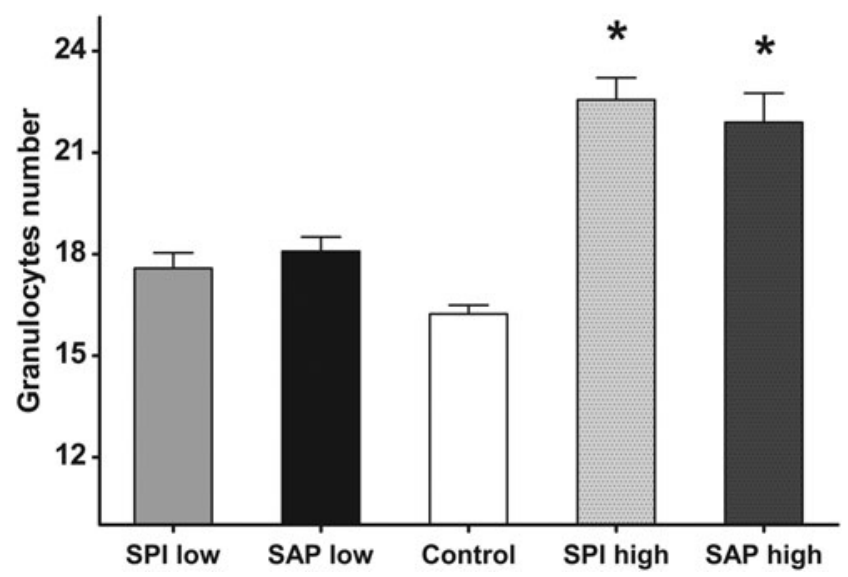

FIG. 2. Increased number of granulocytes in regions of the digestive tract of zebrafish larvae fed SAP or SPI. Quantification of granulocytes in the gastrointestinal tract of zebrafish larvae fed the experimental diets SPI and SAP. For each diet, 120 larvae were assessed in groups containing 20 fish and were fed for 5 days as described previously. The results were derived from the fraction of larvae that ingested the feed. The larvae fed high-SAP or high-SPI showed significant differences in the number of granulocytes with respect to the group of larvae fed the control diet $\left(^{*}\right)$. In contrast, larvae fed low SPI or Low SAP showed no significant differences with respect to the control diet. These results were processed using SPSS 8.0 performing a one-way analysis of variance (ANOVA) to find significant differences among the experimental groups. To determine the significant differences between the groups, Dunnett's multiple comparison was used.
high-SPI diets exhibited markedly increased granulocyte levels and significantly differed from the control group, revealing the important effect of the soy-supplemented components in the diet.

Detection of neutrophils in the digestive tract using Tg(mpx:GFP) ${ }^{i 114}$ line zebrafish. To determine if neutrophils can be localized in the intestinal region in larvae fed different diets, immunohistochemical examination was performed. To make easy the localization of the neutrophils, the transgenic strain $\operatorname{Tg}(m p x: G F P)^{i 114}$ was used. In these larvae neutrophils express GFP under the control of myeloperoxidase promotor, hence these cells can be localized using anti-GFP antibody and revealed by a brown mark. The localization of neutrophils is shown in Figure 3. This figure shows representative sections of larvae fed control (Fig. 3A) or experimental diets (high-SPI, high-SAP; Fig. 3B and C, respectively); in all samples, the brown mark indicating the localization of neutrophils was near to the lumen of the digestive tract. The Figure 3D shows negative samples, where only the secondary antibody was used; in this figure, no brown marks were detected, discarding unspecific reactions. In these immunohistochemical assays, no quantification can be performed; however, these results support that the Sudan black-quantified granulocytes were mostly located in the gut.

\section{Effect of dietary supplementation on the expression of genes related to the innate immune system}

To characterize the effect of the incorporation of high-SAP or high-SPI levels into the diet, several genes related to the innate immune system were assessed by qPCR. Their relative expressions were determined using the larvae fed the control diet as a reference condition; the expression of these genes was compared for the high-SPI- or high-SAP-treated larvae. The expression of cytokines, such as illb and tnfa, was assessed because these proteins are important for the initiation and amplification of inflammatory reactions and constitute important targets for modulating excessive inflammation. In addition, the genes related to the innate immune cells were analyzed with $m p x$ and lyz. The most important granulocytes correspond to the neutrophils, which can be identified by a high number of refractive granules in the cytoplasm, and their activity can be associated with the expression of $m p x$. The lyz gene was previously thought to be macrophage-specific; however, multiple reports indicate that lyz is also expressed in neutrophils. The expression levels of acutephase proteins, including saa and $c 3 b$, which are integral components of the complement system, were analyzed to determine their similarities in terms of their response to infection.

Larvae fed the high-SPI diets exhibited a significant and increased expression of proinflammatory cytokines $i l 1 b$ and tnfa (marked with asterisks in Fig. 4). This result was concurrent with the induction of $m p x$ and saa, which were also significantly different from the control group. In the group fed the high-SAP diet, the expression profile was more complex. The cytokines were not significantly induced. However, illb levels tended to increase while varying widely, and the expression of tnfa remained almost unchanged. Interestingly, the expression of the acute-phase proteins saa and $c 3 b$ was significantly induced, and this result was concurrent with a significant increase in the expression of $m p x$ (marked with stars in Fig. 4). 
FIG. 3. Detection of neutrophils in the digestive tract using $\operatorname{Tg}(\mathrm{mpx}: \mathrm{GFP})^{\mathrm{i} 114}$ line zebrafish. Immunohistochemical examination of representative sections of larvae fed control (A) or experimental diets (high-SPI, high-SAP; $\mathbf{B}$ and $\mathbf{C}$, respectively). (A) Includes an insert remarking the digestive region used in the histological examination. In all samples, the brown mark indicated the localization of neutrophils near to the lumen of the digestive tract. The (D) shows negative sample, where only the secondary antibody was used; in this panel, no brown marks were detected. The brown color is the result of reaction between peroxidase-conjugated antibody and DAB substrate. Toluidine blue was used like ink contrast for the nuclei. m, muscle; pc, pigment cells. Color images available online at www.liebertpub.com/zeb

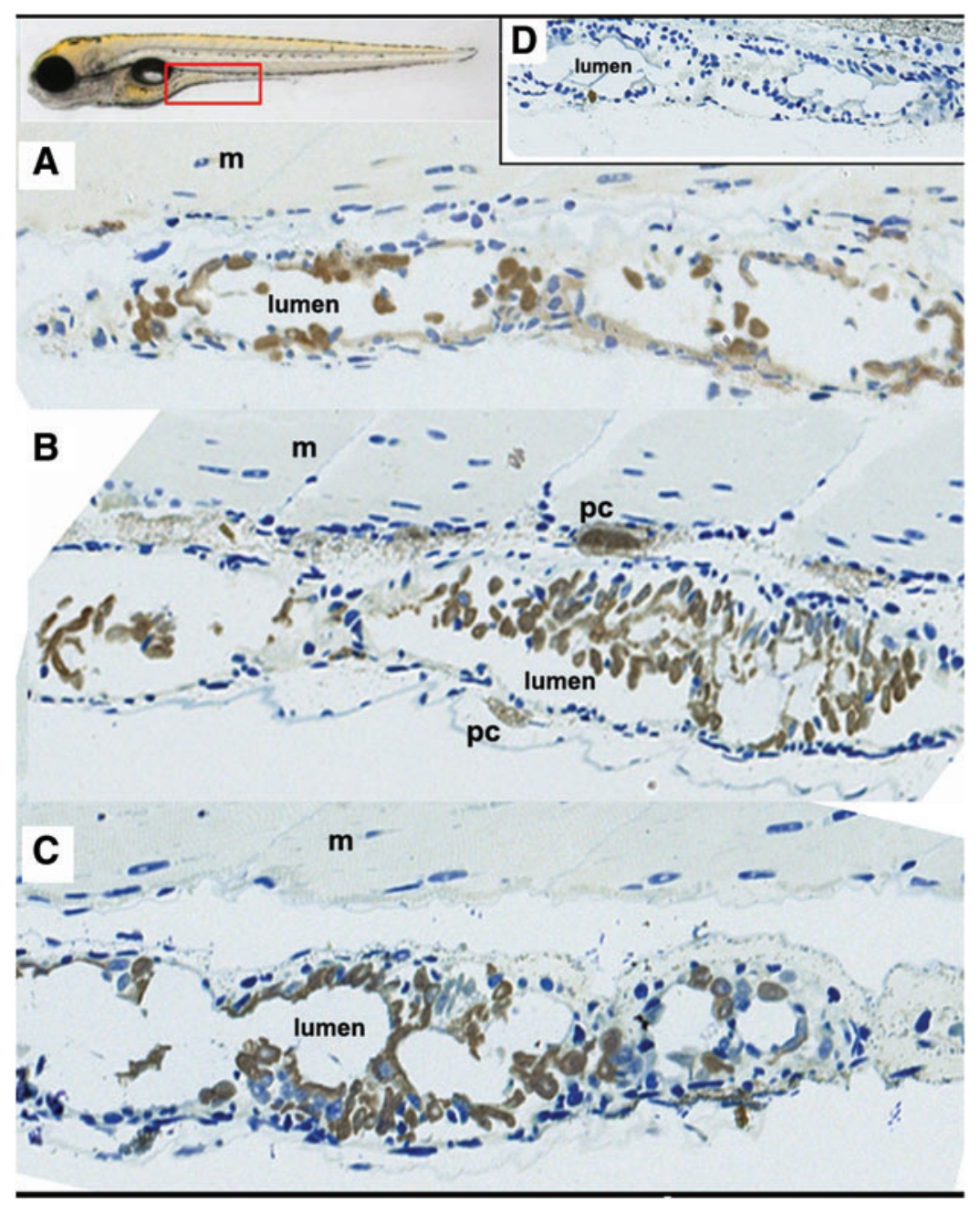

\section{Discussion}

The zebrafish represents a model with unique genetic and in vivo imaging advantages that permit the rapid in vivo analysis of several processes, including developmental digestive physiology and disorders associated with the digestive tract. ${ }^{38,39}$ Despite these advantages and the interest in zebrafish as a model for studies on nutrition or nutrigenomics in other farmed finfish species, its potential as a model organism has not yet been fully realized. ${ }^{40}$

Zebrafish is a stomachless organism and, as in other cyprinids, the uptake of foodstuffs and macromolecules (pinocytosis) occurs in its intestines. ${ }^{41,42}$ Simultaneously, the gut epithelium provides a physical and immunological barrier capable of sensing and responding to microbial incursion or gut lumen antigens. ${ }^{41,42}$ Therefore, we used the zebrafish as a model for inflammatory disorders (enteritis) caused by the inclusion of plant-based ingredients in fishfeed. This is a key issue in understanding the contribution of foodstuffs to the modulation of immunological responses initiated at the level of the gut-associated mucosa. From a nutritional perspective, this knowledge could guide diet formulations to avoid the inflammatory effects of foodstuffs. From a defense point of view, it will be important to modulate the immune system using functional ingredients incorporated into the fishfeed.
In this study, we analyzed the influences of diets supplemented with SBM components (SAP and SPI) on the innate immune system. These findings represent an initial step toward determining the molecular and cellular bases for distal enteritis, a phenomenon described across a broad spectrum of fish, including salmonids, cyprinids, and flatfish. The transparency of the early larval stage of the zebrafish allows for the detection of ingested feed in the gut and also for the visualization of granulocytes near the gut. Our results indicated an increased number of granulocytes associated with the digestive tract in larvae fed high-SPI or high-SAP diets. The observed granulocyte infiltration can be regarded as a sign of gut inflammation because it has been documented that the migration of these cells (mainly neutrophils) to specific epithelia occurs as a response to inflammatory signals. ${ }^{43,44}$ These results were complemented with those from $\operatorname{Tg}(m p x: G F P)^{i 114}$ larvae fed the high-SPI and highSAP diets, among which a specific mark of neutrophils (stained brown) indicated that these granulocytes localized to the gut mucosa. An important aspect of this study is that zebrafish appear to be incapable of mounting an antibody response until early adulthood, 4 weeks postfertilization, before which they rely exclusively on their innate immune system. ${ }^{45}$ Therefore, in the assessed larvae at $10 \mathrm{dpf}$, the observed response to the diets supplemented with SBM components may rely only on the innate immune system. In contrast, most studies have been 


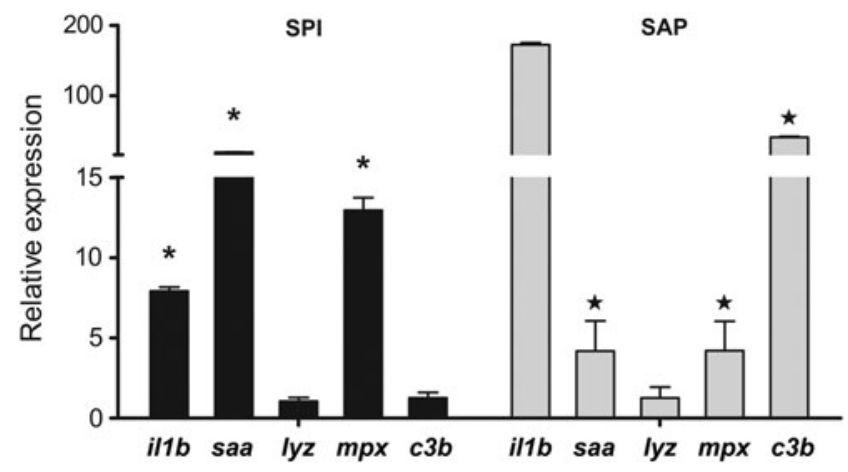

FIG. 4. Induction of genes related to the innate immune system after being fed with SAP or SPI. Graphs of the relative expression of immune genes in zebrafish larvae fed the experimental diets, high-SAP or high-SPI, using a control diet as the reference condition. For each diet, three replicates of 120 larvae were assessed in groups containing 20 fish and were fed for 5 days as described previously. Only larvae that ingested feed were considered for further analysis. On the left-hand side of the graph, the larvae fed with the high-SPI diet showed significantly increased the expression of $i l 1 b$, tnfa, saa, and $\operatorname{mpx}\left({ }^{*} p<0.05\right)$. The genes $l y z$ and $c 3 b$ showed no significant expression difference compared to the control diet. On the right-hand side of the graph, the larvae fed with the high-SAP diet showed significantly increased levels of $s a a, m p x$, and $c 3 b$ expression $\left({ }^{\star} p<0.05\right)$. The genes $i l 1 b, \operatorname{tnfa}$, and lyz showed no significant expression differences compared to the control diet. Each cDNA sample was assessed in quadruplicate (four qPCR), and specificities were validated using melting curves. The relative quantification of each gene was obtained using the Pfaffl method (2002), and the REST 2009 software (Qiagen) used 18S as a reference gene for normalization. qPCR, quantitative polymerase chain reaction.

performed in juveniles or adult fish; hence, the observed effects may not be attributed exclusively to the innate immune system. . $^{15,18,23,46}$

The low-SPI or low-SAP diets corresponded to the supplementation level provided by 30\% SBM dietary inclusion, which is known to induce enteritis in salmonids and common $\operatorname{carp}^{15,18,46}$; however and contrary to our expectations, the zebrafish larvae fed the diets including low quantities of SBM components (low-SAP, low-SPI) demonstrated no increase in the quantity of granulocytes associated with the digestive tract. This finding suggests that in zebrafish larvae a greater amount of SAP or SPI is required to induce the inflammatory signals when presented separately, whereas at low concentrations, probably several SBM components must be present in the diet to induce inflammation. Considering these observations, low-SPI and low-SAP diets were not tested by qPCR analysis. Chen et $a l .{ }^{21}$ reported similar results, noting that the histological structure of the intestine of the Japanese flounder (enteritis-like syndrome) was affected gradually in response to diets supplemented with increasing concentrations of saponins (from 0 to $6.4 \mathrm{~g} / \mathrm{kg}$ ). The effects of dietary saponins in fish have been studied using different plant-based diets. Recently, Chikwati et al. ${ }^{23}$ evaluated SAP supplementation in combination with several plant-based proteins. The authors reported that dietary SAP supplementation at a level expected in diets containing $\sim 20 \%-30 \%$ SBM caused minor-to-moderate effects on the salmon intestinal physiology when added to diets containing maize gluten, sunflower meal, and rapeseed meal. An alternative diet containing pea protein concentrate, saponin supplementation induced major effects similar to the typical effects induced by SBM diets. The increased granulocyte number in larvae fed high-SPI diets suggests that dietary plant-based proteins may be involved in the induction of inflammation signals.

The gene expression profiles observed in larvae fed diets containing high levels of SPI or SAP revealed two important issues. First, these diets induced the upregulation of genes with recognized proinflammatory functions, such as $i l 1 b$, tnfa, and saa, which are involved in the recruitment and migration of granulocytes. ${ }^{47}$ The second issue is the upregulation of proteins involved in defense against infection, such as $m p x$ and $c 3 b$. Larvae fed the diets containing high-SPI exhibited proinflammatory gene expression profiles. It is well known that soy proteins have immunogenic properties. ${ }^{27,28}$ Glycinin and $\beta$-conglycinin, the major storage proteins in soy isolates, have been identified as important food allergens. ${ }^{48}$ Several studies have been conducted to investigate the effects of soybean glycinin and conglycinin on the local and systemic immune responses using various animal models, including mice, swine, and calves. ${ }^{24-26}$ Furthermore, the partial hydrolysis of these proteins by the fermentation of SBM reduces the plasma immunoreactivity in pigs. However, studies conducted in trout suggest that fractions of SBM containing these proteins did not induce alterations in the histology of trout. ${ }^{10,17}$ This discrepancy may be due to the use of diets including low concentrations of soy proteins, which is coincident with our results using the low-SPI diets.

However, larvae fed high-SAP diets exhibited an antiinfection gene expression profile, upregulating proteins involved in the defense against infection, including $m p x$ and $c 3 b$. The enzyme mpx is involved in the production of bactericidal substances in granulocytes. ${ }^{49}$ This induction coincides with the greater number of immune cells in the larvae fed the high-SAP diet. This result may also be related to the stimulation of saa expression. Altogether, these results may be explained by the action of SAPs on the gut. The mechanism by which SAPs trigger the onset of enteritis has been suggested to involve increasing the epithelial permeability, thereby exposing the local immune system to foreign antigens from the gut lumen and leading to an inflammatory response. The foreign antigens that induce inflammation could be antigenic legume proteins or antigens from the intestinal microbiota. Brugman et al. ${ }^{30}$ recently illustrated the influence of microbiota on inflammatory syndromes in the zebrafish gut. They used an oxazolone to induce enterocolitis in zebrafish and added antimicrobials to alter the bacterial populations in the microbiota. Zebrafish dominated by Fusobacteria demonstrated reduced enterocolitis scores. In contrast, the dominance of Proteobacteria reduced eosinophil and lymphocyte infiltration, but the enterocolitis scores were not reduced.

The relation between gene expression and enteritis manifestation has been recently studied. Sahlmann et al. ${ }^{50}$ evaluated multiple time points of the early response in the SBM induced enteritis in the juvenile $S$. salar. These authors used a microarray to describe the intestinal transcriptome and the histological examination to describe the enteritis signals. They showed that the change in the gene expression profile, especially in the immune system set, occurred previous to the 
manifestation of enteritis at the histological level. This finding is coincident with our results in zebrafish larvae, in which we found the increased expression of genes related to the immune system. Subsequently, it is expected that the enteritis symptoms were visualized in longer experimental designs.

In summary, these results indicate that both SAPs and SPIs may play roles in the induction of the inflammatory signals that lead to enteritis in the gut of fish when high levels of these components are incorporated into the diet. This result also supports the use of zebrafish as a model for studies on nutrition or immunomodulation associated with feedstuffs in other farmed finfish species. In particular, this study establishes zebrafish as a model for studying plant-based dietinduced enteritis, which constitutes a major problem in aquaculture.

\section{Acknowledgments}

P.F.-A. was supported by a scholarship from the CONICYT-Chile. R.O. was supported by a scholarship from the Dr. Stekel fellowship from INTA-Nestlé. This investigation was supported by a grant FONDECYT no. 1110253 from CONICYT-Chile.

\section{Disclosure Statement}

No competing financial interests exist.

\section{References}

1. Rawls JF, Samuel BS, Gordon JI. Gnotobiotic zebrafish reveal evolutionarily conserved responses to the gut microbiota. Proc Natl Acad Sci USA 2004;101:4596-4601.

2. Kiron V. Fish immune system and its nutritional modulation for preventive health care. Anim Feed Sci Technol 2012;173: 111-133.

3. Levraud J-P, Colucci-Guyon E, Redd MJ, Lutfalla G, Herbomel P. In vivo analysis of zebrafish innate immunity. Methods Mol Biol 2008;415:337-363.

4. Stevens CE, Hume ID. Contributions of microbes in vertebrate gastrointestinal tract to production and conservation of nutrients. Physiol Rev 1998;78:393-427.

5. Clements KD, Raubenheimer D. Feeding and nutrition. In: The Physiology of Fishes. Evans DH, Claiborne JB (eds), Boca Raton, FL: Taylor and Francis Group, 2006.

6. Oliva-Teles A. Nutrition and health of aquaculture fish. J Fish Dis 2012;35:83-108.

7. Swiatczak B, Rescigno M. How the interplay between antigen presenting cells and microbiota tunes host immune responses in the gut. Semin Immunol 2012;24:43-49.

8. Wacyk J, Powell M, Rodnick K, Overturf K, Hill R, Hardy R. Dietary protein source significantly alters growth performance, plasma variables and hepatic gene expression in rainbow trout (Oncorhynchus mykiss) fed amino acid balanced diets. Aquaculture 2012;356-357:223-234.

9. Sales J. The effect of fish meal replacement by soyabean products on fish growth: a meta-analysis. Br J Nutr 2009;102:1709-1722.

10. Hart SD, Bharadwaj AS, Brown PB. Soybean lectins and trypsin inhibitors, but not oligosaccharides or the interactions of factors, impact weight gain of rainbow trout $(\mathrm{On}$ corhynchus mykiss). Aquaculture 2010;306:310-314.

11. Santigosa E, García-Meilán I, Valentin JM, Pérez-Sánchez J, Médale F, Kaushik S, et al. Modifications of intestinal nutrient absorption in response to dietary fish meal replace- ment by plant protein sources in sea bream (Sparus aurata) and rainbow trout (Onchorynchus mykiss). Aquaculture 2011;317:146-154.

12. Gatlin DM, Barrows FT, Brown P, Dabrowski K, Gaylord TG, Hardy RW, et al. Expanding the utilization of sustainable plant products in aquafeeds: a review. Aquacult Res 2007;38:551-579.

13. Bakke-McKellep AM, Press CM, Baeverfjord G, Krogdahl Å, Landsverk T. Changes in immune and enzyme histochemical phenotypes of cells in the intestinal mucosa of Atlantic salmon, Salmo salar L., with soybean meal-induced enteritis. J Fish Dis 2000;23:115-127.

14. Krogdahl §̊, Bakke-McKellep AM, Baeverfjord G. Effects of graded levels of standard soybean meal on intestinal structure, mucosal enzyme activities, and pancreatic response in Atlantic salmon (Salmo salar L.). Aquacult Nutr 2003;9:361-371.

15. Goncalves AA, Urán PA, Taverne-thiele JJ, Schrama JW, Verreth JA, Rombout JH. Soybean meal induces intestinal inflammation in common carp (Cyprinus carpio L.). Fish Shellfish Immunol 2008;25:751-760.

16. Urán PA, Schrama JW, Rombout JH, Taverne-Thiele JJ, Obach A, Koppe W, et al. Time-related changes of the intestinal morphology of Atlantic salmon, Salmo salar L., at two different soybean meal inclusion levels. J Fish Dis 2009;32:733-744.

17. Knudsen D, Urán P, Arnous A, Koppe W, Frøkiaer H. Saponin-containing subfractions of soybean molasses induce enteritis in the distal intestine of Atlantic salmon. J Agric Food Chem 2007;55:2261-2267.

18. Knudsen D, Jutfelt F, Sundh H, Sundell K, Koppe W, Frøkiaer H. Dietary soya saponins increase gut permeability and play a key role in the onset of soyabean-induced enteritis in Atlantic salmon (Salmo salar L.). Br J Nutr 2008;100:120-129.

19. Sørensen M, Penn M, El-Mowafi A, Storebakken T, Chun-

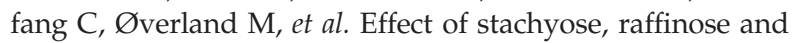
soya-saponins supplementation on nutrient digestibility, digestive enzymes, gut morphology and growth performance in Atlantic salmon (Salmo salar, L). Aquaculture 2011;314:145-152.

20. Krogdahl A, Penn M, Thorsen J, Refstie S, Bakke AM. Important antinutrients in plant feedstuffs for aquaculture: an update on recent findings regarding responses in salmonids. Aquacult Res 2010;41:333-344.

21. Chen W, Ai Q, Mai K, Xu W, Liufu Z, Zhang W, et al. Effects of dietary soybean saponins on feed intake, growth performance, digestibility and intestinal structure in juvenile Japanese flounder (Paralichthys olivaceus). Aquaculture 2011;318: 95-100.

22. Kortner T, Skugor S, Penn MH, Mydland LT, Djordjevic B, Hillestad M, Krasnov A and Krogdahl A. Dietary soyasaponin supplementation to pea protein concentrate reveals nutrigenomic interactions underlying enteropathy in Atlantic salmon (Salmo salar). BMC Vet Res 2012;8:101.

23. Chikwati EM, Venold FF, Penn MH, Rohloff J, Refstie S, Guttvik A, et al. Interaction of soyasaponins with plant ingredients in diets for Atlantic salmon, Salmo salar L. Br J Nutr 2012;107:1570-1590.

24. Guo P, Piao X, Ou D, Li D, Hao Y. Characterization of the antigenic specificity of soybean protein beta-conglycinin and its effects on growth and immune function in rats. Arch Anim Nutr 2007;61:189-200.

25. Sun P, Li D, Dong B, Qiao S, Ma X. Effects of soybean glycinin on performance and immune function in early weaned pigs. Arch Anim Nutr 2008;62:313-321. 
26. $\mathrm{Xu} \mathrm{J}$, Zhou A, Wang Z, Ai D. Effects of glycinin and $\beta$ conglycinin on integrity and immune responses of mouse intestinal epithelial cells. J Ani Plant Sci 2010;20:170-174.

27. Collins S, Desai A, Mansfield G, Hill J, Van Kessel A, Drew $M$. The effect of increasing inclusion rates of soybean, pea and canola meals and their protein concentrates on the growth of rainbow trout: concepts in diet formulation and experimental design for ingredient evaluation. Aquaculture 2012;344-349:90-99.

28. Brandon DL, Friedman M. Immunoassays of soy proteins. J Agric Food Chem 2002;50:6635-6642.

29. Ulloa PE, Iturra P, Neira R, Araneda C. Zebrafish as a model organism for nutrition and growth: towards comparative studies of nutritional genomics applied to aquacultured fishes. Rev Fish Biol Fish 2011;21:649-666.

30. Brugman S, Liu K, Lindenbergh-Kortleve D, Samsom J, Furuta GT, Renshaw SA, et al. Oxazolone-induced enterocolitis in zebrafish depends on the composition of the intestinal microbiota. Gastroenterology 2009;137:1757-1767.

31. Sissener NH, Johannessen LE, Hevrøy EM, Wiik-Nielsen CR, Berdal KG, Nordgreen A, et al. Zebrafish (Danio rerio) as a model for investigating the safety of gm feed ingredients (soya and maize); performance, stress response and uptake of dietary DNA sequences. Br J Nutr 2010;103:3-15.

32. Westerfield M. The Zebrafish Book: A Guide for the Laboratory Use Of the Zebrafish (Danio rerio). Oregon: University of Oregon Press, 1994.

33. Kimmel CB, Ballard WW, Kimmel SR, Ullmann B, Schilling TF. Stages of embryonic development of the zebrafish. Dev Dyn 1995;203:253-310.

34. Haffter P, Granato M, Brand M, Mullins MC, Hammerschmidt M, Kane DA, et al. The identification of genes with unique and essential functions in the development of the zebrafish, Danio rerio. Development 1996;123:1-36.

35. Rønnestad I, Kamisaka Y, Conceição LE, Morais S, Tonheim SK. Digestive physiology of marine fish larvae: hormonal control and processing capacity for proteins, peptides and amino acids. Aquaculture 2007;268:82-97.

36. Renshaw S, Loynes C, Trushell D, Elworthy S, Ingham P and Whyte M. A transgenic zebrafish model of neutrophilic inflammation. Blood 2006;108:3976-3978.

37. Pfaffl MW, Horgan GW, Dempfle L. Relative Expression Software Tool (REST) for group-wise comparison and statistical analysis of relative expression results in real-time PCR. Nucleic Acids Res 2002;30:e36.

38. Fleming A, Jankowski J, Goldsmith P. In vivo analysis of gut function and disease changes in a zebrafish larvae model of inflammatory bowel disease: a feasibility study. Inflamm Bowel Dis 2010;16:1162-1172.

39. Oehlers SH, Flores MV, Okuda KS, Hall CJ, Crosier KE, Crosier PS. A chemical enterocolitis model in zebrafish lar- vae that is dependent on microbiota and responsive to pharmacological agents. Dev Dyn 2011;240:288-298.

40. Kaushik S, Georga I, Koumoundouros G. Growth and body composition of zebrafish (Danio rerio) larvae fed a compound feed from first feeding onward: toward implications on nutrient requirements. Zebrafish 2011;8:87-95.

41. Rombout JH, Abelli L, Picchietti S, Scapigliati G, Kiron V. Teleost intestinal immunology. Fish Shellfish Immunol 2010;31:616-626.

42. Wang Z, Du J, Lam SH, Mathavan S, Matsudaira P. Morphological and molecular evidence for functional organization along the rostrocaudal axis of the adult zebrafish intestine. BMC Genom 2010;11:392.

43. Palic D, Andreasen CB, Ostojic J, Tell RM, Roth JA. Zebrafish (Danio rerio) whole kidney assays to measure neutrophil extracellular trap release and degranulation of primary granules. J Immunol Methods 2007;319:87-97.

44. Walters KB, Green JM, Surfus JC, Yoo SK, Huttenlocher A. Live Imaging of neutrophil motility in a zebrafish model of WHIM syndrome. Blood 2010;116:2803-2811.

45. Herbomel P, Thisse B, Thisse C. Ontogeny and behaviour of early macrophages in the zebrafish embryo. Development 1999;126:3735-3745.

46. Bureau DP, Harris AM, Cho CY. The effects of purified alcohol extracts from soy products on feed intake and growth of chinook salmon (Oncorhynchus tshawytscha) and rainbow trout (Oncorhynchus mykiss). Aquaculture 1998;161:27-43.

47. Boshra H, Li J, Sunyer JO. Recent advances on the complement system of teleost fish. Fish Shellfish Immunol 2006;20:239-262.

48. Heppell LM, Sissons JW, Pedersen HE. A comparison of the antigenicity of soya-bean-based infant formulas. Br J Nutr 1987;58:393-403.

49. Bayne CJ, Gerwick L. The acute phase response and innate immunity of fish. Dev Comp Immunol 2001;25:205-217.

50. Sahlmann C, Sutherland B, Kortner TM, Koop B, Krogdahl $\AA$, Bakke AM. Early response of gene expression in the distal intestine of Atlantic salmon (Salmo salar L.) during the development of soybean meal induced enteritis. Fish Shellfish Immunol 2013;34:599-609.

Address correspondence to: Jaime Romero, PhD Laboratorio de Biotecnología Instituto de Nutrición y Tecnología de los Alimentos (INTA)

Universidad de Chile Santiago 6903625

Chile

E-mail: jromero@inta.cl 